IDDF2018-ABS-0230 TRANSGLUTAMINASE 2 MODULATES INFLAMMATION-ASSOCIATED ANGIOGENESIS VIA VEGFR2 PATHWAY IN CROHN'S DISEASE

Gaoshi Zhou*. Department of Gastroenterology, First Affiliated Hospital of Sun Yat-sen University, China

\subsection{6/gutjnl-2018-IDDFabstracts.33}

Background Angiogenesis is a vital component of the pathogenesis of inflammatory bowel disease(IBD). Vascular endothelial growth factor A(VEGF-A) and its receptors VEGFR2 are the pivotal component of angiogenesis in IBD. Recent studies suggest that transglutaminase 2 regulates angiogenesis in cancer and physiological conditions through VEGFR2 pathway. However, whether transglutaminase 2 are involved in this process of IBD remains elusive.

Methods We use real-time PCR and Western blotting to validate the expression of TGM2 in CD patients' biopsy samples and TNBS-induced murine colitis. Furthermore, we use immunohistochemistry and immunofluorescence of surgical specimen and TNBS/DSS-induced murine colitis section to investigate the location of TGM2 in CD. To investigate the role of TGM2 in CD, human intestinal microvascular endothelial cells (HIMEC) and mouse intestinal microvascular endothelial cells (MIMEC) was treated with inflammatory cytokines. Finally, TNBS murine colitis model was treated with IL-9 neutralising antibody and weight loss, intestine length, as well as microvessel density, was evaluated after treatment.

Results We found that the TGM2 was up-regulated in colonic mucosa of CD patients and TNBS-induced colitis. In addition, TGM2 expression mainly locates in microvessel. (figure 1) Furthermore, after treating with IL-9, TGM2 and VEGFR2 were significantly upgraded in HIMEC and MIMEC. In contrary, weight loss, intestine length, as well as microvessel density in TNBS murine colitis model, was significantly improved after treating with IL-9 neutralising antibody.
Conclusions Our findings provide new evidence that TGM2 plays an important role in angiogenesis in CD, thus TGM2 may represent a novel therapeutic target for IBD.

\section{IDDF2018-ABS-0231 ACIDOSIS PROMOTES THE STEMNESS OF CANCER STEM CELLS THROUGH VITAMIN D-VITAMIN D RECEPTOR SIGNALLING PATHWAY IN COLORECTAL CANCER}

Peishan Hu*, Ting Li, Huaiqiang Ju, Ruihua Xu. Sun Yat-sen University Cancer Center, State Key Laboratory of Oncology in South China, China

\subsection{6/gutjnl-2018-IDDFabstracts.34}

Background The tumour recurrence, metastasis and treatment resistance of colorectal carcinoma (CRC) are closely related to acidic tumour microenvironment and colorectal cancer stem cells (colorectal CSCs). Here we identified the regulatory effects of the acidic tumour microenvironment on vitamin D receptor (VDR) and the regulation of the signal axis on the self-renewal of colorectal stem cells.

Methods Using western blotting and relative quantitative PCR to detect VDR expression in 43 pairs of normal and CRC samples and acidic condition. Self-renewal ability of colorectal CSCs was detected by sphere formation assay, limited dilution assay and flow cytometry. Chromatin immunoprecipitation, gain of function and lose of function assay was used to screen the target genes of VDR and their effects on colorectal CSCs. Results Firstly we found VDR mRNA decreased under acidic condition. And the expression of VDR in CRC tissue compared to adjacent normal tissue was decreased significantly. In addition, the VDR protein expression was the lowest in colorectal CSCs compared to normal, and CRC cells, VDR expression in colorectal cancer cells with strong metastatic ability was also reduced. Over-expressing VDR in CRC sample cell line resulted in decreased cell migration and invasion ability. The proportion of PROM1 (prominin 1) positive cells decreased when over-
A

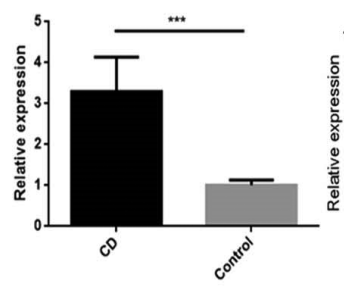

C

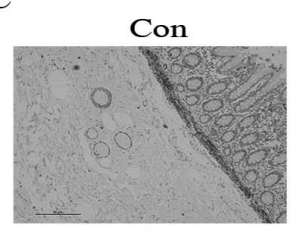

Con

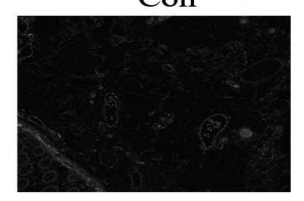

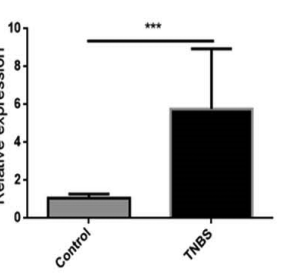

CD

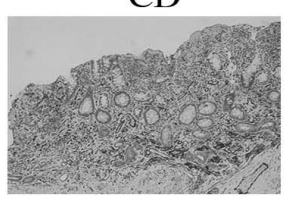

CD

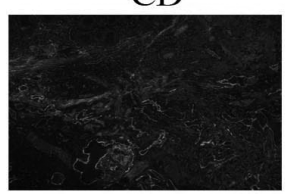

B
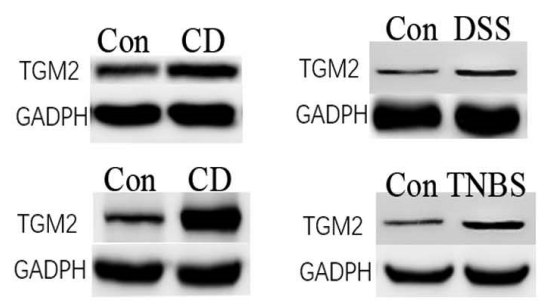

D
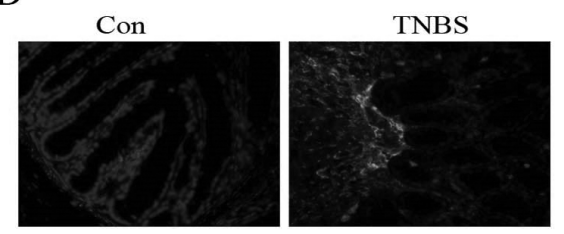

Con

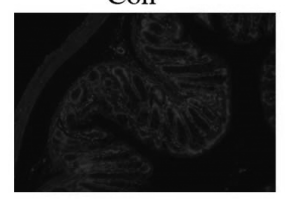


expressed VDR in colorectal CSCs, the number and size of spheres formed by cells were markedly reduced and the sphere cells differentiated and generated processes. While knockdown of VDR in CRC cells resulted in the elevated proportion of PROM1 positive cells, increased number and size of spheres and elevated expression of SOX2 (SRY-box 2). We also found that VDR was combined with the promoter region of SOX2 and POU5F1 (POU class 5 homeobox 1), which contained vitamin $\mathrm{D}$ response elements, in a series of stem cell markers.

Conclusions Acidic tumour microenvironment may regulate vitamin D signalling pathway by lowering the expression of VDR. VDR inhibited the invasion, metastasis and cancer stem cell phenotype of CRC cells through interacting with the promoter of SOX2 and POU5F1. Suggesting normalising tumour microenvironment and combined use of vitamin D and chemotherapeutic drugs are a potentially effective strategy in the treatment of CRC.

\section{IDDF2018-ABS-0233 N6-ADENOSINE METHYLTRANSFERASE METTL3 PROMOTES TUMOUR METASTASIS VIA SOX2 MRNA M6A MODIFICATION IN COLORECTAL CARCINOMA}

Ting Li*, Peishan Hu, Zhixiang Zuo, Huaiqiang Ju. Sun Yat-sen University Cancer Center, State Key Laboratory of Oncology in South China, China

\subsection{6/gutjnl-2018-IDDFabstracts.35}

Background Colorectal carcinoma(CRC) is one of the most common malignant tumours, which main cause of death is tumour metastasis. The role of METTL3 (N6-adenosine-methyltransferase $70 \mathrm{kDa}$ subunit) in cancer has not been well studied. This study reveals a new role of METTL3 in promoting CRC tumour progress through m6A modification of SOX2 (SRY-box 2) and influencing the Epithelial-Mesenchymal Transition process.

Methods Western blot and Immunohistochemistry were used to detect METTL3 expression in cell lines and patient's tissues. The MTS assay, invasion assay, sphere formation assay was performed to detect the function of METTL3. Cell based xenograft model and PDX model revealed the clinical benefits of target METTL3 in vivo. Transcriptome sequencing and uencing were used to screen the m6A target genes, and MeRIP qPCR was used to detect the m6A level of the target gene.

Results Using TCGA database, we found that METTL3 was significantly upregulated in human CRC and high expression of METTL3 in metastasis CRC was associated with poor prognosis of patients. Knockdown of METTL3 in CRC sample cell line and SW620 dramatically suppressed cellular proliferation, migration and sphere formation in vitro. METTL3 inhibition suppresses CRC tumorigenesis and metastasis in both cell models and PDX models in vivo. Mechanistically, we performed RNA-sequencing and MeRIP-sequencing in SW480 and SW620 CRC cell lines and SOX2 was identified as the target of METTL3-mediated m6A modification. The protein level, m6a modification level, and RNA stability of SOX2 was significantly decreased upon METTL3 inhibition. Comparing with the m6a-site-mutant SOX2, only the wildtype modified SOX2 promoting tumour progress through activating EMT process. The RNA and protein level of SOX2 downstream genes were dramatically diminished after METTL3 knockdown and both SOX2 and its target genes showed a positive correlation with METTL3 expression in CRC patients.

Conclusions In summary, we demonstrated that METTL3, acting as an oncogene, was frequently upregulated in human CRC and contributed to CRC metastasis. METTL3 maintained SOX2 expression to activate EMT process through the m6A dependent mechanism. Thus, our findings reveal an important role of METTL3 and provide a potential target of treatment in colorectal carcinoma.

\section{IDDF2018-ABS-0237 EFFECT OF LAUGHTER YOGA AND CLAPPING EXERCISE ON RENAL FUNCTIONS AND QUALITY OF LIFE IN PATIENTS SUFFERING FROM CHRONIC KIDNEY DISEASE IN SOUTH DELHI METRO POPULATION}

Vinod Sharma*. Shri Maha Maya Vaishnav Devi Mandir Research Institute, Dept of Applied Sciences, New Delhi, India

\subsection{6/gutjnl-2018-IDDFabstracts.36}

Background Chronic kidney disease (CKD) is a worldwide public health problem and emerging health problem in India. To study new methods of CKD rehabilitation complications by laughter yoga and clapping exercise program in south Delhi metro population.

Methods For this purpose, we used laughter yoga, which includes respiratory laughing and fun exercises. Fifty-six patients (subject) between 45-65 years old with CKD were studied and divided into two groups (laughter yoga and clapping exercise group and control group) to see the effect of laughter yoga and clapping exercise group in CKD for 30 days. Using a cross-sectional design, which includes age, family history of CKD patients, exercise status and waist circumference, fasting glucose and insulin, glucose tolerance test (GTT), renal ultrasonography, regular monitoring of blood pressure, renal function, requirement of a number of dialysis, serum creatinine level and glycosylated haemoglobin (HbA1c) and quality of life (QOL) indicators were done CKD patients. A $30 \mathrm{~min}$ lecture was followed by $30 \mathrm{~min}$ intense clapping workout for those participants who had laughter yoga included in the program.

Results The present study showed that after three months treatment there was a significant reduction of systolic and diastolic blood pressure, blood glucose levels, glycosylated haemoglobin levels, significant reduction in blood urea and serum creatinine levels, and significant improvement in physical and psychological domain compare to normal levels with changes in lifestyle. Present study highlight that the successful treatment of CKD patients not only requires drugs; but also family care, lifestyle education, harmonised mind-body-soul, awareness, psychological support, preventive approach toward activity of daily living.

Conclusions Our study indicated the importance of daily opportunities for laughter in patients with CKD patients. For subjects in yoga and exercise group, the need for dialysis was less when compared to control group although this difference was statistically insignificant. Therefore, preventive CKD education programme and promotion of laughter yoga and clapping will be the future plan of action for control and treatment. 